\title{
Patients with marginal zone dural lymphoma successfully treated with rituximab and bendamustine: A report of two cases
}

\author{
YUTAKA TSUTSUMI $^{1}$, SHINICHI ITO ${ }^{1}$, JUN NAGAI ${ }^{1}$, TAKAHIRO TATENO $^{1}$ and TAKANORI TESHIMA ${ }^{2}$ \\ ${ }^{1}$ Department of Hematology, Hakodate Municipal Hospital, Hakodate, Hokkaido 041-8680; \\ ${ }^{2}$ Department of Hematology, Hokkaido University Graduate School of Medicine, Sapporo, Hokkaido 060-8638, Japan
}

Received September 6, 2020;Accepted June 8, 2021

DOI: $10.3892 / \mathrm{mco} .2021 .2371$

\begin{abstract}
Primary dural low-grade marginal zone B-cell lymphoma of mucosa-associated lymphoid tissue (MALT) is a rare disease whose main treatment has been local surgery or radiotherapy. Until now, there have been no cases of dural MALT lymphoma treatment of a patient with several relapses or systemic disease. The present study included two patients with dural MALT lymphoma who had several relapses or systemic disease. Since local therapy was not enough to control the disease for these patients, they were treated with systemic chemotherapy. The patients were administered rituximab $\left(375 \mathrm{mg} / \mathrm{m}^{2}\right)$ and two days of bendamustine $\left(90 \mathrm{mg} / \mathrm{m}^{2}\right)$. Both patients recovered from their clinical symptoms immediately, and their tumors were reduced. During and after rituximab and bendamustine therapy, no central nervous system (CNS) metastasis or cerebrospinal fluid invasion of MALT were observed. The current approach using rituximab and bendamustine treatment was effective against dural MALT lymphoma and may prevent its invasion of the CNS.
\end{abstract}

\section{Introduction}

Dural lymphoma is a rare subtype of primary central nervous system lymphoma (PCNSL) found in the dura mater that differs biologically from types of PCNSLs that are usually high-grade, diffuse large B-cell lymphomas $(1,2)$. Dural lymphoma is usually a low-grade marginal zone B-cell lymphoma of mucosa-associated lymphoid tissue (MALT). MALT lymphoma responds well to local therapies such as surgery and radiation therapy. Most patients recover completely and have good local disease control in response to this treatment, but the risk of systemic relapse appears to be high, as the dura mater is outside of the blood-brain barrier (BBB) (2). Previous reports

Correspondence to: Dr Yutaka Tsutsumi, Department of Hematology, Hakodate Municipal Hospital, 1-10-1 Minato, Hakodate, Hokkaido 041-8680, Japan

E-mail: yutsutsu@shore.ocn.ne.jp

Key words: dural mucosa-associated lymphoid tissue, bendamustine, central nervous system showed that systemic chemotherapy was mainly high-dosage methotrexate treatment (HD-MTX) together with radiation therapy or surgery (3). Because the dura mater is outside of the BBB, HD-MTX may not be useful for treating dural MALT lymphomas. In this report, we cover two dural MALT lymphoma patients: One with systemic lymphoid swelling and one who experienced a third relapse after local radiation therapy and surgery. These two patients went into complete remission after treatment with rituximab and bendamustine.

\section{Case report}

Case 1. A 65-year-old man was urgently transported to a neurosurgical hospital on May 19, 2019 with a generalized seizure. A space-occupying lesion with midline deviation was found in the left frontal region of his brain (Fig. 1A), and a craniotomy tumor removal was performed on May 21. The tumor was distributed in layers in the subcranial subcutaneous tissue with a hematoma inside and outside the dura mater. Hematoxylin and eosin staining was was performed according to routine hospital procedures. The pathological diagnosis of the tumor was MALT lymphoma (Fig. 1B). Although flow cytometry was positive for CD20, CD19, cell surface kappa, and HLA-DR, and G-band pattern showed poor proliferation, fluorescence in situ hybridization (FISH) was negative for BIRC3-MALT1 translocation. Moreover, the patient was in the MALT Lymphoma Clinical Stage IVA IPI High-Intermediate risk group. Rituximab was used in combination for systemic therapy, and on July 17, 2019, treatment was started with rituximab $375 \mathrm{mg} / \mathrm{m}^{2}$ (Day 1) and bendamustine $90 \mathrm{mg} / \mathrm{m}^{2}$ (Days 2 and 3: R-Bendamustine). Methotrexate $12 \mathrm{mg}$ and prednisolone $10 \mathrm{mg}$ were only administered intrathecally the first time (because the cytodiagnosis was Class I). Six courses of the same regimen were administered thereafter. Symptoms such as headaches and seizures quickly improved with the first treatment, and by the time treatment was completed, his disability had also improved. The lesions shrunk (Fig. 1C) and are currently being monitored.

Case 2. A 55-year-old man underwent a medical checkup in June 2010, and a computed tomography (CT) scan showed a parasternal tumor and axillary lymphadenopathy. In July of the same year, a sternum tumor and right axillary lymph node biopsy was performed, and marginal zone B-cell lymphoma was diagnosed. 

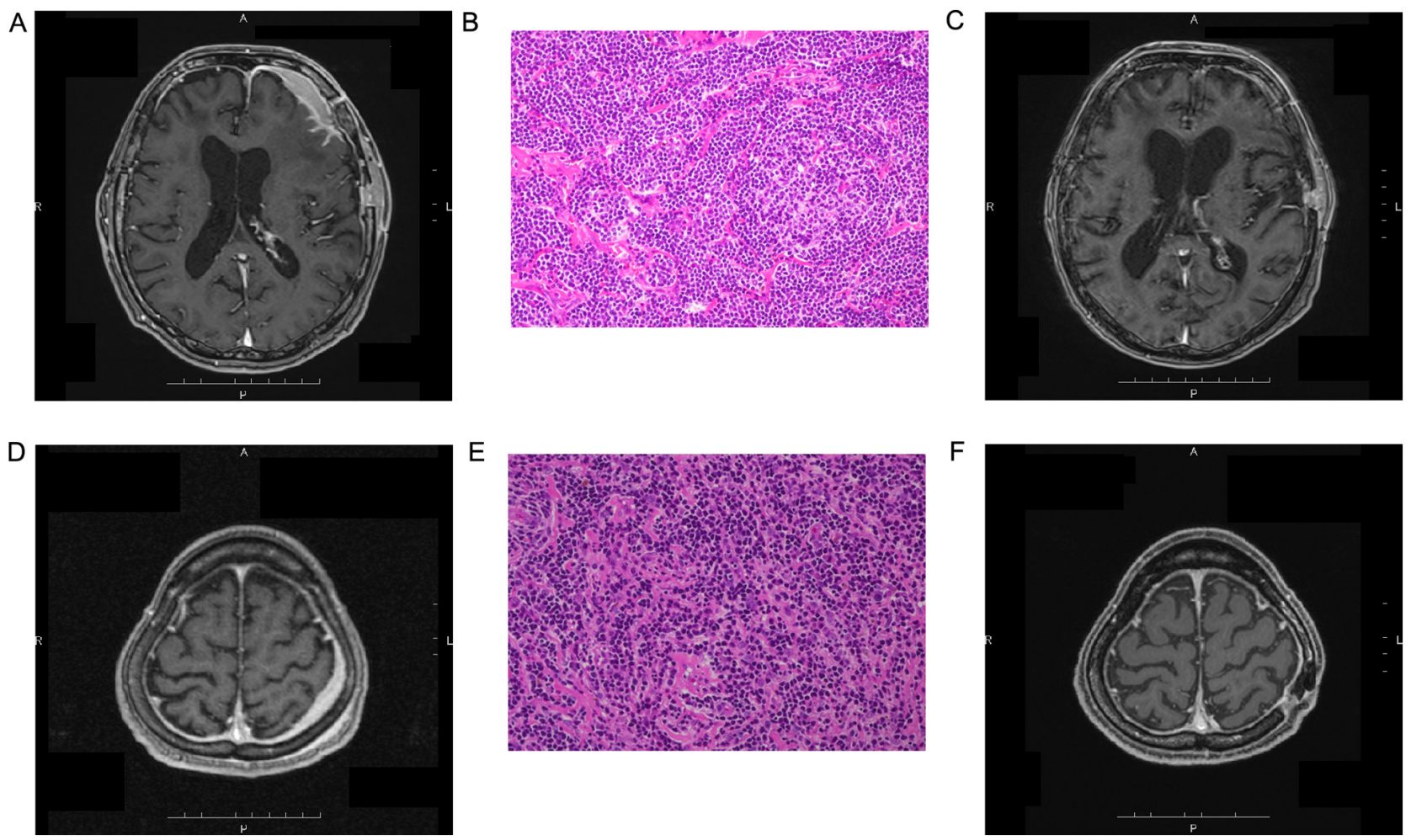

Figure 1. (A) MRI image shows that the tumor was distributed in layers in the subcranial subcutaneous tissue with a hematoma inside and outside the dura mater. (B) Small- to medium-sized lymphoid cell proliferation was observed in the dura mater (magnification, x20). (C) The tumor went into remission after treatment with R-Bendamustine. (D) MRI image with contrast enhancement shows a thickening of the dura. (E) Small- to medium-sized lymphoid cells infiltrated the dura mater (magnification, x20). (F) Dural thickening disappeared after treatment with R-Bendamustine.

At the first visit, his immunoglobulin $\mathrm{M}(\operatorname{IgM})$ was $1,981 \mathrm{mg} / \mathrm{dl}$

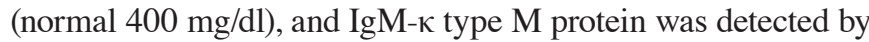
immunoelectrophoresis. Since Helicobacter pylori (H. pylori) was detected in his stomach in August 2010, H.pylori was eradicated with clarithromycin $400 \mathrm{mg}$, amoxicillin $1500 \mathrm{mg}$, and a proton pump inhibitor in anticipation of lymphoma reduction without any effect. Thus, on Day 1 , rituximab $375 \mathrm{mg} / \mathrm{m}^{2}$ was administered; on Day 2, cyclophosphamide $750 \mathrm{mg} / \mathrm{m}^{2}$, doxorubicin $50 \mathrm{mg} / \mathrm{m}^{2}$, and vincristine $1.4 \mathrm{mg} / \mathrm{m}^{2}$ were administered; on Days 2-6, prednisolone $60 \mathrm{mg}$ /day was administered. However, no symptoms such as fever or general fatigue were observed. IgM did not decrease at all and was determined to be unaffected. In September 2010, the symptoms improved from daily administration of thalidomide $100 \mathrm{mg}$ and prednisolone $10 \mathrm{mg}$, but the sternum tumor did not shrink. In November, radiation therapy was performed at $40 \mathrm{~Gy} / 20 \mathrm{Fr}$ on the sternum tumor and bilateral axilla. The tumor shrunk, and the thalidomide was discontinued. In October 2014, a CT scan revealed a soft mass in the left heart diaphragm angle. In December 2014, a thoracoscopic longitudinal tumor resection was performed, and a recurrence of a marginal zone lymphoma was diagnosed. Flow cytometry was positive for CD20, CD19, cell surface kappa, and HLA-DR. G-band pattern showed no chromosomal abnormalities, and FISH was negative for BIRC3-MALT1 translocation. Since then, we have been following the patient after re-administration with prednisolone to maintain remission. In July 2016, interleukin-2 receptor (IL-2R) and IgM increased again, but the chest and abdominal CT scan showed no new lesions. However, an image with contrast enhancement showed thickening on the left parietal lobe in the head MRI performed in August 2016 (Fig. 1D). A dural biopsy was performed in September 2016. Hematoxylin and eosin staining was performed according to routine hospital procedures. Small- and medium-sized lymphocytes were diffused in the dura mater tissue (Fig. 1E), and it was determined that a marginal zone lymphoma had recurred. Since the patient did not desire a cerebrospinal fluid (CSF) injection, rituximab was used in combination for systemic therapy. Four courses of R-Bendamustine were administered, and the dural lesion disappeared (Fig. 1F). In February 2019, positron emission tomography was performed and detected multiple bone metastases. R-Bendamustine was used, but the patient stopped using it because he was allergic to it. After that, four courses of FCM therapy (Day 1: Mitoxantrone $8 \mathrm{mg} / \mathrm{m}^{2}$; Days 1-3: Fludarabine $25 \mathrm{mg} / \mathrm{m}^{2}$ and Cyclophosphamide $200 \mathrm{mg} / \mathrm{m}^{2}$ ) were performed, and remission was achieved. The patient is currently undergoing follow-up, and the dural lesion has not recurred during the course.

\section{Discussion}

Although dura mater is outside of the BBB, the status of dural lymphomas is confused with that of PCNSL. Almost all dural lymphomas are MALT lymphomas, so almost all treatments involve surgery and radiotherapy (4). Chemotherapy is used for CNS lymphomas, so treatment almost always involves regimens with HD-MTX (4). Bustoros et al analyzed previous reports to determine prognosis for dural MALT lymphoma and reported that the five-year overall survival rate was $96.7 \%$ and the progression-free survival (PFS) rate was $81.2 \%$, while de la Fuente et al reported that the three-year and five-year PFS rates were 89 and $76 \%$, respectively $(4,3)$. However, from a developmental point 
of view, the CNS originates at the neural tube or ectoderm, while the dura mater originates at the neural crest or mesoderm, wherein the dura mater is not considered to be nerve tissue or a central nerve. In fact, de la Fuente et al reported that almost all patients received surgery and radiotherapy (3). Thus, HD-MTX may be an excessive treatment against dural MALT lymphoma.

In this report, two cases of dural MALT lymphoma were treated with rituximab and bendamustine. One patient relapsed three times and received refractory treatment of R-CHOP, bortezomib, and thalidomide therapy. A dural tumor with systemic MALT lymphoma was discovered. Both patients had systemic MALT, so focal radiation therapy and surgery were insufficient. Our rituximab and bendamustine therapy was effective on both patients and prevented CNS metastasis or CSF invasion of MALT from occurring afterwards. Both cases were Class I in early CSF cytology with no meningeal dissemination, which may be why treatment with rituximab and bendamustine therapy was effective and administration of CSF methotrexate or high-dose methotrexate is possibly unnecessary. On the other hand, the possibility of dural lesions directly infiltrating the CNS cannot be ruled out, therefore, mass methotrexate therapy cannot be ruled out for cases with meningeal invasion (2).

Rituximab and bendamustine therapy is an effective treatment for MALT lymphoma (5). There was an $88 \%$ possibility of event-free survival being four years in this study. Kim et al reported the effectiveness of rituximab, bendamustine, vincristine, cytarabine, and dexamethasone (R-BOAD) against PCNSL. Although the CSF concentration of bendamustine was only $0.025 \%$, they reported tumor regression (6). Furthermore, Li et al analyzed the CNS penetration of bendamustine and found that bendamustine penetrated $35 \%$ of the CNS in a mouse model (7). These reports suggest that bendamustine was effective against both systemic and CNS MALT lymphomas. Some reports showed the effectiveness of bendamustine in treatments during the refractory period or after recurrence of the PCNSL with patients who were treated with HD-MTX (8-10).

In conclusion, although there have only been a few cases thus far, rituximab and bendamustine treatment appears to be effective against dural MALT lymphomas and may prevent invasion of the CNS. Further study is necessary to clarify its clinical efficacy against dural MALT lymphoma.

\section{Acknowledgements}

Not applicable.

\section{Funding}

No funding was received.

\section{Availability of data and materials}

All data generated or analyzed during this study are included in this published article.

\section{Authors' contributions}

YT, SI and JN were clinical investigators for the case. YT drafted the manuscript. TTa contributed to acquisition of data, and TTe contributed to analysis and interpretation of data, and both participated in writing or technical editing of the manuscript. YT and SI confirm the authenticity of all the raw data. All authors read and approved the final version of the manuscript.

\section{Ethics approval and consent to participate}

The study was approved by the local Ethics Committee of Hakodate Municipal Hospital Institutional Review Board (approval no. 2019-47; Hakodate, Japan).

\section{Patient consent for publication}

Written informed consent was obtained from the patients described in the report. A copy of the consent may be requested from the corresponding author.

\section{Competing interests}

The authors declare that they have no competing interests.

\section{References}

1. Ayanambakkam A, Ibrahimi S, Bilal K and Cherry MA: Extranodal marginal zone lymphoma of central nervous system. Clin Lymphoma Myeloma Leuk 18: 34-37.e8, 2018.

2. Iwamoto FM and Abrey LE: Primary dural lymphoma: A review. Neurosurg Focus 21: E5, 2006.

3. de la Fuente MI, Haggiagi A, Moul A, Young RJ, Sidani C, Markoe A, Vega F, DeAngelis LM and Lossos IS: Marginal zone dural lymphoma: The memorial sloan kettering cancer center and university of miami experience. Leuk Lymphoma 58: 882-888, 2017.

4. Bustoros M, Liechty B, Zagzag D, Liu C, Shepherd T, Gruber D, Raphael B and Placantonakis DG: A rare case of composite dural extranodal marginal zone lymphoma and chronic lymphocytic leukemia/small lymphocytic lymphoma. Front Neurol 9: 267, 2018.

5. Salar A, Domingo-Domenech E, Panizo C, Nicolas C, Bargay J, Muntanola A, Canales M, Bello JL, Sancho JM, Tomas JF, et al: First-line response-adapted treatment with the combination of bendamustine and rituximab in patients with mucosa-associated lymphoid tissue lymphoma (MALT2008-01): A multicentre, single-arm, phase 2 trial. Lancet Haematol 1: e104-e111, 2014.

6. Kim T, Choi HY, Lee HS, Jung SH, Ahn JS, Kim HJ, Lee JJ, Yoo HD and Yang DH: Clinical response and pharmacokinetics of bendamustine as a component of salvage $\mathrm{R}-\mathrm{B}(\mathrm{O}) \mathrm{AD}$ therapy for the treatment of primary central nervous system lymphoma (PCNSL). BMC Cancer 18: 729, 2018.

7. Li Z, Caulfield T, Qiu Y, Copland JA and Tun HW: Pharmacokinetics of bendamustine in the central nervous system: Chemoinformatic screening followed by validation in a murine model. Med Chem Commun 3: 1526-1530, 2012.

8. Lim T, Kim SJ, Kim K, Lee JI, Lim DH, Lee DJ, Baek K, Lee HY, Han B, Uhm JE, et al: Primary CNS lymphoma other than DLBCL: A descriptive analysis of clinical features and treatment outcomes. Ann Hematol 90: 1391-1398, 2011.

9. Chamberlain MC: Salvage therapy with bendamustine for methotrexate refractory recurrent primary CNS lymphoma: A retrospective case series. J Neurooncol 118: 155-162, 2014.

10. Renfrow JJ, Detroye A, Chan M, Tatter S, Ellis T, McMullen K, Johnson A, Mott R and Lesser GJ: Initial experience with bendamustine in patients with recurrent primary central nervous system lymphoma: A case report. J Neurooncol 107: 659-663, 2012. International (CC BY-NC-ND 4.0) License. 